WS10-A03

\title{
Surface Microseismic Monitoring of Hydraulic Fracturing of a Shale-Gas Reservoir Using Low- Frequency Sensors
}

\author{
H. Zhang* (University of Science and Technology of China), X. Zeng \\ (University of Wisconsin-Madison), X. Zhang (University of Science and \\ Technology of China), Y. Liu (University of Science and Technology of \\ China) \& Y. Zhang (Beijing MiSeis Technologies)
}

\section{SUMMARY}

In this study, we report results from a surface microseismic monitoring of a 21 -stage hydraulic fracturing of a shale gas reservoir along a horizontal well. The depths of hydraulic fracturing range from $2500 \mathrm{~m}$ to $3020 \mathrm{~m}$. A surface array consisting of 45 sensors was deployed around the well, with 27 L22E short-period and $18 \mathrm{CMG}-6 \mathrm{TD}$ broadband seismometers. For analyzing the continuously recorded microseismic data, we adopted the following steps: (1) event detection, (2) event location, (3) event focal mechanism determination, and (4) microseismic imaging. The double-difference seismic tomography method of Zhang and Thurber [2003] is applied to determine the Vp and Vs anomalies due to the fracturing process. In total, more than 700 events were detected. The events can be categorized into two groups: group I near the wellbore and group II about 500 meters to the east of the wellbore. The group II events are likely induced by the pore pressure increase directly caused by fluid migrated from the fracturing spot or the pressure perturbation caused by undrained response of fluid injection. The velocity tomography shows that the fracturing process causes lower Vp and Vs anomalies around the fracturing zone. 


\section{Amsterdam '14}

\section{Introduction}

China has great potentials in shale gas reservoirs but is still at the early stage of shale gas development. For shale gas development, hydraulic fracturing is needed to create fractures within shale reservoirs so that gas has passages to move. The accurate knowledge of spatial extent of fractures is necessary for reservoir engineers to evaluate whether fracturing is appropriate and the potential product of the field. During fracturing, microseismic events generally occur due to stress perturbations along weak zones, i.e. fractures; therefore microseismic locations can be used to characterize fracture distribution.

In this study, we report results from a surface microseismic monitoring of a multiple-stage hydraulic fracturing of a shale gas reservoir along a horizontal well. The study region is located in southwest China where many pilot wells have been drilled to assess the shale gas development potential in China (Figure 1). There are 21 fracturing stages along the $1400 \mathrm{~m}$ horizontal well. The depths of hydraulic fracturing range from $2500 \mathrm{~m}$ to $3020 \mathrm{~m}$ (shadowed zone in Figure 1a). According to the well logging data, limestone is the main rock type for the segment above $1300 \mathrm{~m}$ while mudstone is the major rock type below $1300 \mathrm{~m}$. The target layer is composed of shale and clay. We used low frequency and high sensitivity geophones to monitor induced seismicity by hydraulic fracturing. A surface array consisting of 45 sensors was deployed around the well, with 27 L22E short-period and 18 CMG-6TD broadband seismometers (Figure 1b).

\section{Method}

For analyzing the continuously recorded microseismic data, we adopted the following steps:

(1) Event detection. A characteristic function is constructed to detect the weak microseismic events, which reflects the amplitude and frequency variations at the phase arrival.

(2) Event location. A migration-based full waveform location method is developed to automatically locate microseismic events. For events with relatively high signal-to-noise ratio, $\mathrm{P}$ and $\mathrm{S}$ first arrivals are picked and the double-different location method of Waldhauser and Ellsworth [2000] is applied to refine event locations.

(3) Event focal mechanism determination: The polarities of the first-arrival $\mathrm{P}$ waves are used to determine event focal mechanisms and the corresponding compressive axes.

(4) Microseismic imaging. The double-difference seismic tomography method of Zhang and Thurber [2003] is applied to determine the Vp and Vs models around the fracturing depth. The shear-wave splitting analysis is also used to analyze the seismic anisotropy of the fracturing zone.

\section{Examples}

In total, more than 700 events were detected during 21 stages, among which 154 events with at least 8 picks are selected for focal mechanism analysis (Figure 2a). The events can be categorized into two groups: group I near the wellbore and group II about 500 meters to the east of the wellbore. Magnitudes of most events range from -2 to -1 and the two groups have similar focal mechanism distributions (Figure $2 b$ ). The azimuths of compressive $\mathrm{P}$ axes are shown in a polar histogram (Figure 2c). Most P-axis azimuths fall into the NW-NNE direction, which is consistent with the regional tectonic stress direction. The group I events are likely directly caused by the fracturing process while the group II events are likely induced by the pore pressure increase directly caused by fluid migrated from the fracturing spot or the pressure perturbation caused by undrained response of fluid injection.

\section{Conclusions}

Our study shows that with a relatively "sparse" surface array it is also possible to detect and locate small magnitude events $(\mathrm{M}<-1)$ induced by hydraulic fracturing. Therefore for monitoring hydraulic fracturing in some regions with favorable surface conditions and relatively shallow reservoir zones, it is possible to use a sparse surface array. 


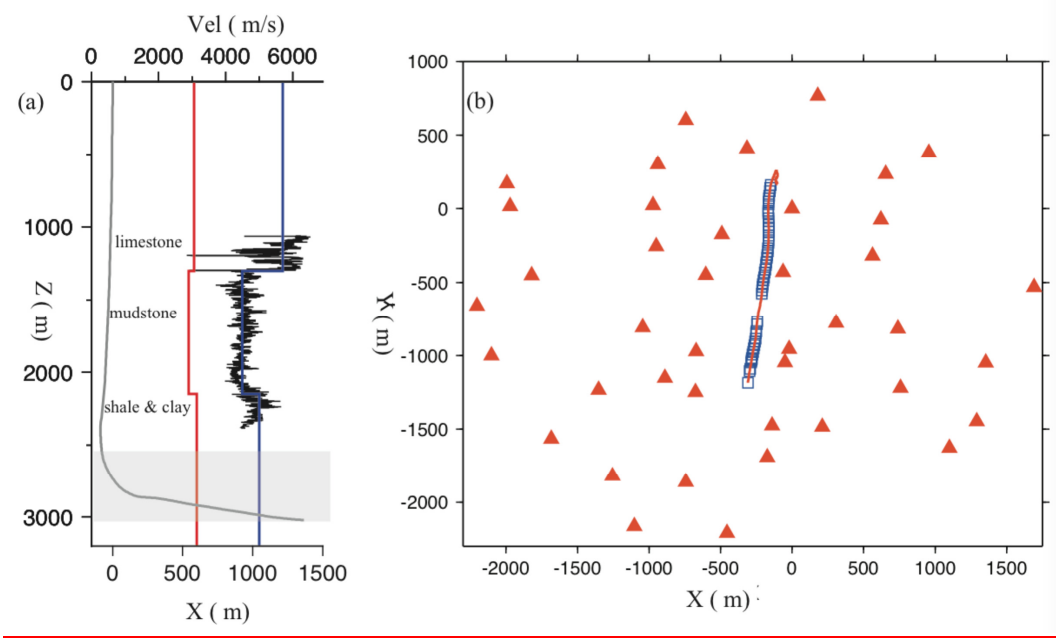

Figure 1 (a) Layered Vp (blue line) and Vs (red line) velocity models derived from sonic logging velocity curve (black) as well as horizontal well trajectory (gray line). (b) Surface monitoring array (triangles) and horizontal well trajectory (red line) with perforation shot locations (squares).
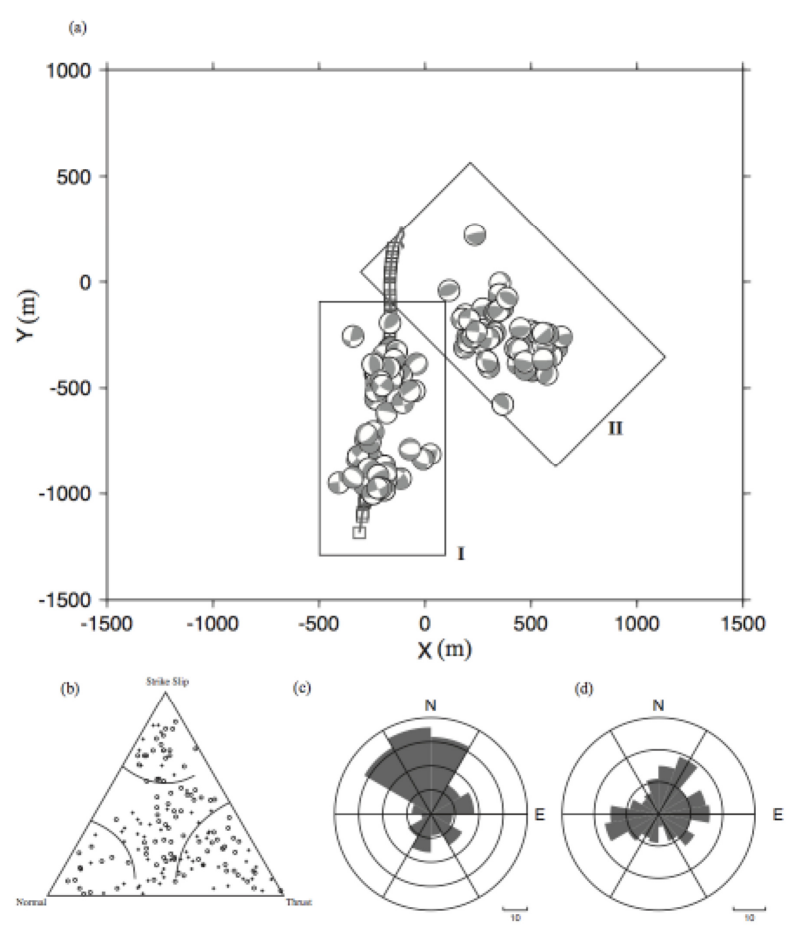

Figure 2 (a) Map view of focal mechanisms; (b) Triangle diagram of focal mechanisms. Circle and cross denote events from group I and II; (c) Distribution of azimuths of $P$ axes.

\section{References}

Waldhauser, F. and Ellsworth, W. L. [2000] A double-difference earthquake location algorithm: Method and application to the northern Hayward fault, California. Bulletin of the Seismological Society of America, 90, 1353-1368.

Zhang, H. and Thurber, C. H. [2003] Double-difference tomography: The method and its application to the Hayward fault, California. Bulletin of the Seismological Society of America, 93, 1875-1889. 